\title{
On 3D minimal massive gravity
}

\section{Mohsen Alishahiha, ${ }^{a, b}$ Mohammad M. Qaemmaqami, ${ }^{c}$ Ali Naseh ${ }^{c}$ and Ahmad Shirzad ${ }^{d, c}$}

${ }^{a}$ School of Physics, Institute for Research in Fundamental Sciences (IPM), P.O. Box 19395-5531, Tehran, Iran

${ }^{b}$ Theory Group, Physics Department, CERN, CH-1211 Geneva 23, Switzerland

${ }^{c}$ School of Particles and Accelerators, Institute for Research in Fundamental Sciences (IPM), P.O. Box 19395-5531, Tehran, Iran

${ }^{d}$ Department of Physics, Isfahan University of Technology, P.O. Box 84156-83111, Isfahan, IRAN

E-mail: alishah@ipm.ir, m.qaemmaqami@ipm.ir, naseh@ipm.ir, shirzad@ipm.ir

ABSTRACT: We study linearized equations of motion of the newly proposed three dimensional gravity, known as minimal massive gravity, using its metric formulation. By making use of a redefinition of the parameters of the model, we observe that the resulting linearized equations are exactly the same as that of TMG . In particular the model admits logarithmic modes at critical points. We also study several vacuum solutions of the model, specially at a certain limit where the contribution of Chern-Simons term vanishes.

Keywords: Classical Theories of Gravity, Gauge-gravity correspondence, Chern-Simons Theories

ArXiv ePrint: 1409.6146 


\section{Contents}

1 Introduction 1

2 Linearized analysis in metric formalism 3

$\begin{array}{llc}3 & \text { Wave solutions } & 6\end{array}$

$\begin{array}{llr}4 & \text { On new MMG vacuum solutions } & 8\end{array}$

$\begin{array}{lll}5 & \text { Logarithmic CFT and new anomaly } & 10\end{array}$

6 Conclusions 12

\section{Introduction}

Minimal Massive Gravity (MMG) [1] is a three dimensional massive gravity which has the same minimal local structure as Topologically Massive Gravity (TMG) [2]. Working within the "Chern-Simons-like" formulation of massive gravity [3] one may define the action of the MMG model from that of TMG by adding an extra term. Adding this extra term would open up the possibility to get a consistent ghost free and non-tachyonic three dimensional theory. Nevertheless its linearization about a flat or AdS vacuum still has a single massive mode.

To explore this point better, let us first consider the TMG model in the Chern-Simons like formulation whose Lagrangian 3-form may be written as follows [1]

$$
L_{\mathrm{TMG}}=-\sigma e \cdot R+\frac{\Lambda_{0}}{6} e \cdot e \times e+h \cdot T(\omega)+\frac{1}{2 \mu}\left(\omega \cdot d \omega+\frac{1}{3} \omega \cdot \omega \times \omega\right),
$$

where $\sigma$ is a sign, $\Lambda_{0}$ is a primary cosmological constant, $e$ and $\omega$ are dreibein and dualised spin-connection, respectively. $\mu$ is a mass parameter of the model.

In terms of these variables the Lorentz covariant torsion and curvature 2-forms are given by

$$
T(\omega)=d e+\omega \times e, \quad R(\omega)=d \omega+\frac{1}{2} \omega \times \omega .
$$

In the action (1.1) the auxiliary field $h$ may be thought of as a Lagrange multiplier that imposes the zero torsion constraint.

It is then straightforward to find equations of motion of the above action by taking the variation with respect to the fields $h, e$ and $\omega$

$$
T(\omega)=0, \quad \sigma R(\omega)-\frac{\Lambda_{0}}{2} e \times e-D(\omega) h=0, \quad R(\omega)+\mu e \times h+\sigma \mu T(\omega)=0 .
$$

For generic values of $\Lambda_{0}$ and $\mu$, these equations admit several solutions including AdS, BTZ and warped AdS black holes solutions [4-7]. It is, however, known that this model suffers from the fact that the energy of graviton and the mass of BTZ black holes cannot be positive at the same time; tuning the parameters to get a positive energy for the graviton 
would result in BTZ solutions with negative mass, and vice versa, i.e a positive mass BTZ black hole gives a negative energy for graviton. Alternatively, if one thinks of the model as one which provides a gravitational dual for a two dimensional conformal field theory (CFT), the corresponding CFT would have negative central charge whenever the propagating spin-2 mode in the bulk has positive energy.

In order to have a well defined unitary model, it was proposed in [8] that the parameters of the TMG may be tuned to particular values (critical points), so that the modes with negative mass are removed from the model leading to a gravitational theory which could provide a holographic description for a chiral CFT. It was, however, observed [9] that although at the "critical points" the modes with negative mass are removed from the theory, the model admits new "logarithmic" modes which again results in a non-unitary theory. ${ }^{1}$

To circumvent the above problem, the authors of [1], proposed a new model, named MMG, whose Lagrangian 3-form is

$$
L_{\mathrm{MMG}}=L_{\mathrm{TMG}}+\frac{\alpha}{2} e \cdot h \times h .
$$

Then, it was shown that in the specific range of the parameters of the model, it is possible to get positive central charges for the dual conformal field theory and, at the same time to have a graviton with positive energy.

The equations of motion derived from the action of MMG are given by [1]

$$
T(\omega)-\alpha e \times h=0, \quad R(\omega)+\mu e \times h+\sigma \mu T(\omega)=0,
$$

and

$$
-\sigma R(\omega)+\frac{\Lambda_{0}}{2} e \times e+D(\omega) h+\frac{\alpha}{2} h \times h=0 .
$$

Defining $\Omega=\omega+\alpha h$ one can solve the equations in (1.5) to find $\Omega$ and $h$ in terms of $e$. Then plugging the results into the equation (1.6) one can find an equation for the metric as follows

$$
\bar{\sigma} G_{\mu \nu}+\bar{\Lambda}_{0} g_{\mu \nu}+\frac{1}{\mu} C_{\mu \nu}+\frac{\gamma}{\mu^{2}} J_{\mu \nu}=0
$$

where the Einstein tensor $G_{\mu \nu}$, and the Cotton tensor $C_{\mu \nu}$ are given by

$$
G_{\mu \nu}=R_{\mu \nu}-\frac{1}{2} R g_{\mu \nu}, \quad C_{\mu \nu}=\epsilon_{\mu}{ }^{\alpha \beta} \nabla_{\alpha}\left(R_{\beta \nu}-\frac{1}{4} g_{\beta \nu} R\right)
$$

while the curvature-squared symmetric tensor $J_{\mu \nu}$ is defined by ${ }^{2}$

$$
J_{\mu \nu}=R_{\mu}{ }^{\alpha} R_{\alpha \nu}-\frac{3}{4} R R_{\mu \nu}-\frac{1}{2} g_{\mu \nu}\left(R^{\alpha \beta} R_{\alpha \beta}-\frac{5}{8} R^{2}\right) .
$$

Here the parameters $\gamma, \bar{\sigma}$ and $\bar{\Lambda}_{0}$ are defined in terms of the parameters of the action as follows $^{3}$

$$
\gamma=\frac{\alpha}{(1+\sigma \alpha)^{2}}, \quad \bar{\sigma}=-\left(\sigma+\alpha+\frac{\alpha^{2} \Lambda_{0}}{2 \mu^{2}(1+\sigma \alpha)^{2}}\right), \quad \bar{\Lambda}_{0}=-\Lambda_{0}\left(1+\sigma \alpha-\frac{\alpha^{3} \Lambda_{0}}{4 \mu^{2}(1+\sigma \alpha)^{2}}\right) .
$$

\footnotetext{
${ }^{1}$ For logarithmic solution in higher dimensional gravity see, e.g. [10-13].

${ }^{2}$ In our convention, $\epsilon^{123}=\frac{1}{\sqrt{-g}}$ and $\epsilon_{123}=-\sqrt{-g}$.

${ }^{3}$ Since our convention for Levi-Civita tensor differs from that in [1] by a minus sign, we will get an extra overall minus sign in our following equations.
} 
It is argued in [1] that the equation (1.7) for $\alpha \neq 1$ cannot be obtained from any conventional deformation of the TMG action with higher order curvature terms.

It is the aim of this article to further study the MMG model. In particular we will reconsider the linearization of MMG around an AdS vacuum solution in the metric formulation of the model. We will see that the resulting equations are exactly the same as that of TMG upon a redefinition of the parameters of the model. This shows that TMG and MMG have locally the same structure, though when it comes to computing the conserved charges they behave differently. This is, indeed, the reason why MMG could provide a well defined three dimensional gravity with minimal degrees of freedom. We shall also see that there is a point in the moduli space of parameters of the model, where the linearized equations of motion reduce into that of 3 dimensional conformal gravity.

We will also study wave solutions of the MMG model, where one observes that, just like the TMG model, there are critical points at which the model exhibits logarithmic solutions, though unlike the TMG case, a priori one is not forced to be at the critical points. Having found a logarithmic solution for particular values of the parameters of the theory, it is then natural to propose that the MMG model could also provide a holographic description for a logarithmic CFT (LCFT) at the critical point. Following this idea we will also compute the new anomaly of the corresponding LCFT.

We shall also study other possible solutions of the MMG model, specially those with zero contribution from the Chern-Simons term. Motivated by these solutions we will study the model in a limit where $\mu \rightarrow \infty, \gamma \rightarrow \infty$, while keeping $\kappa=\frac{\gamma}{\mu^{2}}$ fixed. In this limit the Chern-Simons terms drops though the curvature-squared term survives the limit. Note that in the Chern-Simons like formulation of MMG, this limit corresponds to a limit in which $\mu \sim \frac{1}{\epsilon}, 1+\sigma \alpha \sim \epsilon$ for $\epsilon \rightarrow 0$.

The paper is organized as follows. In the next section we will study the linearization of MMG in the metric formulation where we will show that the model has several critical points at which it becomes either conformal, or admits logarithmic modes. In section three we study wave solutions of the model where we will find logarithmic solutions at the critical points. In section four we will study new solutions of the MMG equations of motion specially in the limit where the contribution of Chern-Simons term vanishes. Clearly the obtained solutions cannot be solutions of TMG, nor Einstein gravity. In section five we will compute the new anomaly of the corresponding dual LCFT. The last section is devoted to conclusions.

\section{Linearized analysis in metric formalism}

In this section, using the metric formulation of MMG given by the equation (1.7), we study linear excitations of metric around an $\mathrm{AdS}_{3}$ vacuum. Denoting the vacuum metric by $\bar{g}_{\mu \nu}$, one sets

$$
g_{\mu \nu}=\bar{g}_{\mu \nu}+h_{\mu \nu}
$$

where $h_{\mu \nu}$ is the small perturbation and $\bar{g}_{\mu \nu}$ satisfies the following equation

$$
\bar{R}_{\mu \nu}=-\frac{2}{l^{2}} \bar{g}_{\mu \nu}, \quad \text { with } \quad l^{2}=-\frac{1}{2 \mu} \frac{1}{\bar{\Lambda}_{0}}\left(\mu \bar{\sigma} \pm \sqrt{\mu^{2} \bar{\sigma}^{2}-\bar{\Lambda}_{0} \gamma}\right) .
$$


At the linearized level, the equation of motion (1.7) reduces to ${ }^{4}$

$\bar{\sigma}\left(R_{\mu \nu}^{(1)}-\frac{1}{2} \bar{g}_{\mu \nu} R^{(1)}+\frac{3}{l^{2}} h_{\mu \nu}\right)+\bar{\Lambda}_{0} h_{\mu \nu}+\frac{1}{\mu} \epsilon_{\mu}{ }^{\alpha \beta} \bar{\nabla}_{\alpha}\left(R_{\beta \nu}^{(1)}-\frac{1}{4} \bar{g}_{\beta \nu} R^{(1)}+\frac{2}{l^{2}} h_{\beta \nu}\right)=\frac{-\gamma}{2 \mu^{2} l^{2}} J_{\mu \nu}^{(1)}$,

where

$$
J_{\mu \nu}^{(1)}=R_{\mu \nu}^{(1)}-\frac{1}{2} \bar{g}_{\mu \nu} R^{(1)}+\frac{5}{2 l^{2}} h_{\mu \nu} .
$$

Moreover the linearized Ricci tensor and Ricci scalar are given by

$$
\begin{aligned}
& R_{\mu \nu}^{(1)}=\frac{1}{2}\left(-\bar{\nabla}^{2} h_{\mu \nu}-\bar{\nabla}_{\mu} \bar{\nabla}_{\nu} h+\bar{\nabla}_{\mu} \bar{\nabla}_{\sigma} h_{\nu}^{\sigma}+\bar{\nabla}_{\nu} \bar{\nabla}_{\sigma} h_{\mu}^{\sigma}+\frac{2}{l^{2}} \bar{g}_{\mu \nu} h-\frac{6}{l^{2}} h_{\mu \nu}\right), \\
& R^{(1)}=-\bar{\nabla}^{2} h+\bar{\nabla}_{\rho} \bar{\nabla}_{\sigma} h^{\rho \sigma}+\frac{2}{l^{2}} h,
\end{aligned}
$$

with $h \equiv \bar{g}^{\mu \nu} h_{\mu \nu}$. Using these expressions and in the gauge $\bar{\nabla}^{\mu} h_{\mu \nu}=\bar{\nabla}_{\nu} h$ the equation $(2.3)$ reads

$$
\begin{aligned}
\left(\bar{\sigma}+\frac{\gamma}{2 \mu^{2} l^{2}}\right)(- & \left.\frac{1}{2} \bar{\nabla}^{2} h_{\mu \nu}+\frac{1}{2} \bar{\nabla}_{\mu} \bar{\nabla}_{\nu} h-\frac{3}{l^{2}} h_{\mu \nu}\right)+\left(\frac{3 \bar{\sigma}}{l^{2}}+\bar{\Lambda}_{0}+\frac{5}{4} \frac{\gamma}{\mu^{2} l^{4}}\right) h_{\mu \nu} \\
& +\frac{1}{\mu} \epsilon_{\mu}{ }^{\alpha \beta} \bar{\nabla}_{\alpha}\left(-\frac{1}{2} \bar{\nabla}^{2} h_{\beta \nu}+\frac{1}{2} \bar{\nabla}_{\nu} \bar{\nabla}_{\beta} h+\frac{1}{2 l^{2}} \bar{g}_{\beta \nu} h-\frac{1}{l^{2}} h_{\beta \nu}\right)=0 .
\end{aligned}
$$

On the other hand from the trace of the equation (2.6) one finds

$$
\left(4 \bar{\Lambda}_{0}-\frac{\gamma}{\mu^{2} l^{4}}\right) h=0
$$

which for $\left(4 \bar{\Lambda}_{0}-\frac{\gamma}{\mu^{2} l^{4}}\right) \neq 0$, sets the trace of the perturbation, $h$, to zero. By making use of the linearized Bianchi identity and setting $h=0$, the equation (2.6) may be recast into the following form

$$
\left(\bar{\nabla}^{2}+\frac{\gamma-4 \mu^{2} l^{4} \bar{\Lambda}_{0}}{l^{2}\left(\gamma+2 \mu^{2} l^{2} \bar{\sigma}\right)}\right) h_{\mu \nu}+\frac{2 \mu l^{2}}{\gamma+2 \mu^{2} l^{2} \bar{\sigma}}\left(\bar{\nabla}^{2}+\frac{2}{l^{2}}\right) \epsilon_{\mu}{ }^{\alpha \beta} \bar{\nabla}_{\alpha} h_{\beta \nu}=0 .
$$

Furthermore using the relation between $l$ and $\bar{\Lambda}_{0}$ given in the equation (2.2) one arrives at

$$
\left(\bar{\nabla}^{2}+\frac{2}{l^{2}}\right)\left(\frac{1}{\tilde{\mu}} \epsilon_{\mu}{ }^{\alpha \beta} \bar{\nabla}_{\alpha} h_{\beta \nu}+h_{\mu \nu}\right)=0, \quad \text { with } \quad \tilde{\mu}=\frac{\gamma+2 \mu^{2} l^{2} \bar{\sigma}}{2 \mu l^{2}} .
$$

Note that in this notation the mass of the massive graviton is

$$
M^{2}=\frac{\tilde{\mu}^{2} l^{2}-1}{l^{2}} .
$$

It is interesting to note that this equation is exactly the same as that found from the linearization of TMG around an AdS vacuum (see the equation 29 [8]), with the replacement of $\mu$ with $\tilde{\mu}$. This, in turn, shows that locally TMG and MMG have the same degrees of freedom as emphasised in [1]. Note that for $\gamma=0$ one has $\tilde{\mu}=-\sigma \mu$.

\footnotetext{
${ }^{4}$ Here and in what follows, the covariant derivative is defined with respect to the background metric $\bar{g}_{\mu \nu}$.
} 
Having realized the similarity between the linearized equation of MMG and that of TMG one can utilize the results of [8] to find the spectrum of the perturbation of MMG around an AdS vacuum. Indeed, following [8] one may rewrite the linearized equation (2.9) in terms of the quadratic Casimir operators of the $\mathrm{SL}(2, \mathbb{R})_{L} \times \mathrm{SL}(2, \mathbb{R})_{R}$ algebra associated with the isometry group of the AdS background geometry as follows [8]

$$
\left[2\left(L^{2}+\bar{L}^{2}\right)+3+\tilde{\mu}^{2} l^{2}\right]\left[L^{2}+\bar{L}^{2}+2\right] h_{\mu \nu}=0 .
$$

Therefore the solutions of the linearized equation can be classified by the representation of $\mathrm{SL}(2, \mathbb{R})_{L} \times \mathrm{SL}(2, \mathbb{R})_{R}$ algebra. More precisely consider primary states $\left|\psi_{\mu \nu}\right\rangle$ with weight $(h, \bar{h})$, so that

$$
\begin{array}{rlrl}
L_{0}\left|\psi_{\mu \nu}\right\rangle & =h\left|\psi_{\mu \nu}\right\rangle, & & L_{1}\left|\psi_{\mu \nu}\right\rangle=0, \\
\bar{L}_{0}\left|\psi_{\mu \nu}\right\rangle=\bar{h}\left|\psi_{\mu \nu}\right\rangle, & & \bar{L}_{1}\left|\psi_{\mu \nu}\right\rangle=0 .
\end{array}
$$

Thus, in the present case the primary weights $(h, \bar{h})$ satisfy the following equation

$$
\left[2 h(h-1)+2 \bar{h}(\bar{h}-1)-3-\tilde{\mu}^{2} l^{2}\right][h(h-1)+\bar{h}(\bar{h}-1)-2]=0 .
$$

Here we have used the fact that

$$
L^{2}\left|\psi_{\mu \nu}\right\rangle=-h(h-1)\left|\psi_{\mu \nu}\right\rangle, \quad \bar{L}^{2}\left|\psi_{\mu \nu}\right\rangle=-\bar{h}(\bar{h}-1)\left|\psi_{\mu \nu}\right\rangle
$$

Note also that from the gauge condition one has $h-\bar{h}= \pm 2$.

The equation (2.13) has two branches of solutions where either of its factors is zero

$$
\begin{aligned}
& \text { Branch 1: }\left(h^{(0)}, \bar{h}^{(0)}\right)= \begin{cases}\left(\frac{3}{2} \pm \frac{1}{2},-\frac{1}{2} \pm \frac{1}{2}\right), & h^{(0)}-\bar{h}^{(0)}=2, \\
\left(-\frac{1}{2} \pm \frac{1}{2}, \frac{3}{2} \pm \frac{1}{2}\right), & h^{(0)}-\bar{h}^{(0)}=-2,\end{cases} \\
& \text { Branch } 2:\left(h^{(m)}, \bar{h}^{(m)}\right)= \begin{cases}\left(\frac{3}{2} \pm \frac{1}{2} \tilde{\mu} l,-\frac{1}{2} \pm \frac{1}{2} \tilde{\mu} l\right), & h^{(m)}-\bar{h}^{(m)}=2, \\
\left(-\frac{1}{2} \pm \frac{1}{2} \tilde{\mu} l, \frac{3}{2} \pm \frac{1}{2} \tilde{\mu} l\right), & h^{(m)}-\bar{h}^{(m)}=-2 .\end{cases}
\end{aligned}
$$

As one observes, the spectrum is exactly the same at that of the TMG model. In particular for normalizable modes where one has to keep the upper signs in the above equation, the first branch corresponds to left and right moving massless gravitons: $(2,0),(0,2)$, while from the second branch and taking into account that the equation is invariant under $\tilde{\mu} \rightarrow-\tilde{\mu}$, one gets normalizable modes corresponding to the massive gravitons $\left(\frac{3}{2}-\frac{1}{2} \tilde{\mu} l,-\frac{1}{2}-\frac{1}{2} \tilde{\mu} l\right)$.

Although unlike the TMG model, a priori, we are not forced to fix the parameter $\tilde{\mu} l$, one could still have critical points $\tilde{\mu} l= \pm 1$ at which the massive gravitons degenerate with either of left or right massless gravitons. In terms of the original parameters of the model in the metric formulation the critical points are given by

$$
\gamma=-2 \mu l(\mu l \bar{\sigma} \mp 1)
$$

while in terms of the parameters appearing in the Chern-Simons-like formalism, using the equation (1.10), they occur at

$$
\alpha=-2\left(\sigma \pm \frac{1}{\mu l}\right)
$$


Since the linearized equations of motion for MMG has locally the same form as TMG, one may wonder if the model reduces to a three dimensional conformal gravity in the limit of $\tilde{\mu} \rightarrow 0$. Indeed form the definition of $\tilde{\mu}$ one observes that $\tilde{\mu}=0$ corresponds to $\gamma=-2 \mu^{2} l^{2} \bar{\sigma}$. On the other hand at this point the coefficients of the first and second terms in the equation (2.6) vanishe, yielding to

$$
\frac{1}{\mu} \epsilon_{\mu}{ }^{\alpha \beta} \bar{\nabla}_{\alpha}\left(-\frac{1}{2} \bar{\nabla}^{2} h_{\beta \nu}+\frac{1}{2} \bar{\nabla}_{\nu} \bar{\nabla}_{\beta} h+\frac{1}{2 l^{2}} \bar{g}_{\beta \nu} h-\frac{1}{l^{2}} h_{\beta \nu}\right)=0,
$$

which is just the contribution from the Cotton tensor. In other words, for this special value of $\gamma$ the theory of MMG, at the linearized level, has locally the same content as that of 3D conformal gravity.

\section{$3 \quad$ Wave solutions}

In the previous section we have seen that the MMG model has an AdS vacuum and small perturbations around this vacuum have the same structure as that of TMG. Since the TMG model, besides the AdS vacuum, has other vacuum solutions, it is then natural to look for other solutions of the MMG model too. In particular one may study wave solutions in this model. ${ }^{5}$ To proceed we consider an ansatz for a wave solution as follows

$$
g_{\mu \nu}=\bar{g}_{\mu \nu}+F k_{\mu} k_{\nu}
$$

with $k_{\mu}$ being a null vector with respect to the background AdS metric $\bar{g}_{\mu \nu}$ which could be parametrized as

$$
d s^{2}=\frac{l^{2}}{r^{2}}\left(d r^{2}-2 d u d v\right)
$$

where $l$ is given in equation (2.2) and $u, v$ are light-like coordinates. With this notation, our ansatz for the wave solution takes the following form

$$
d s^{2}=\frac{l^{2}}{r^{2}}\left(d r^{2}-2 d u d v-F(r, u) d u^{2}\right) .
$$

Here $F(r, u)$ is an arbitrary function which can be determined by equations of motion. By substituting this ansatz into the equations of motion (1.7), one finds

$$
\frac{1}{4 \mu^{2} l^{2} r}\left[-2 \mu l r^{2} \frac{\partial^{3} F}{\partial r^{3}}+\left(2 \mu^{2} l^{2} \bar{\sigma}+\gamma\right)\left(r \frac{\partial^{2} F}{\partial r^{2}}-\frac{\partial F}{\partial r}\right)\right]=0 .
$$

Using the definition of $\tilde{\mu}$ given in the equation (2.9), the above differential equation may be recast into the following form

$$
-r^{2} \frac{\partial^{3} F}{\partial r^{3}}+\tilde{\mu} l\left(r \frac{\partial^{2} F}{\partial r^{2}}-\frac{\partial F}{\partial r}\right)=0
$$

which can be solved to give a generic AdS-wave solution with

$$
F(r, u)=F_{0}(u)+F_{2}(u) r^{2}+F_{\tilde{\mu}}(u) r^{1+\tilde{\mu} l} .
$$

\footnotetext{
${ }^{5}$ The wave solutions of TMG and NMG with logarithmic profile have been first studied in [14, 15].
} 
It is then obvious that the solution degenerates at the critical points $\tilde{\mu} l= \pm 1$, as expected. Indeed, just like TMG, one would expect that the MMG model exhibits logarithmic solutions at the critical points. In fact at these points one gets

$$
\begin{array}{llll}
\tilde{\mu} l=-1 & (\text { or } \gamma=-2 \mu l(\mu l \bar{\sigma}+1)) & \rightarrow & F(r, u)=\tilde{F}_{0}(u) \log (r)+F_{0}(u)+F_{2}(u) r^{2} \\
\tilde{\mu} l=1 & (\text { or } \gamma=-2 \mu l(\mu l \bar{\sigma}-1)) & \rightarrow & F(r, u)=F_{0}(u)+F_{2}(u) r^{2}+\tilde{F}_{2}(u) r^{2} \log (r) .
\end{array}
$$

It is important to note that at the first critical point where $\tilde{\mu} l=-1$, the model admits a solution which is not asymptotically locally AdS geometry. In other words in the context of Gauge/Gravity duality, the leading log-term corresponds to turning on an irrelevant operator in the dual field theory. Actually one would expect that the dual field theory to be a LCFT. On the other hand at the second critical point where $\tilde{\mu} l=1$, the geometry is, indeed, asymptotically locally AdS, though, comparing with an AdS geometry, its field theory dual is now in a new vacuum.

We note also that at $\tilde{\mu}=0$ the solution generates a linear term in the expression of $F$

$$
F(r, u)=F_{0}(u)+F_{1}(u) r+F_{2}(u) r^{2},
$$

indicating that the corresponding gravity should come from a 3D conformal gravity, as anticipated in the previous section. Note that since $k_{\mu}$ is a null vector, the first order perturbation given by $h_{\mu \nu}=F k_{\mu} k_{\nu}$ is, indeed, an exact solution of the whole equations of motion.

Note also that setting the integration constants $F_{0}$ and $F_{2}$ in the solution (3.6) to zero and assuming $\tilde{\mu} l<-1$ one finds the three dimensional Schrödinger solution (null solution) with dynamical scaling $z=\frac{1}{2}(1-\tilde{\mu} l)$

$$
d s^{2}=l^{2}\left(\frac{d r^{2}}{r^{2}}-\frac{2 d v d u}{r^{2}}-\frac{d u^{2}}{r^{2 z}}\right) .
$$

Null solutions in TMG and NMG have also been studied in [6] and [16], respectively.

It is worth mentioning that one could have found the wave solutions and the corresponding critical points from the Chern-Simons like formulation of MMG. Actually for our ansatz (3.3) one has

$$
e^{1}=\frac{l}{r} d r, \quad e^{2}=\frac{1}{2} F(r, u) d u+d v, \quad e^{3}=\frac{l^{2}}{r^{2}} d u,
$$

which can be used to read the dual spin-connection from the torsion-less condition

$$
\Omega_{r}^{1}=-\frac{1}{r}, \quad \Omega_{u}^{2}=\frac{1}{2 l}\left(-r \frac{\partial F}{\partial r}+F\right), \quad \Omega_{u}^{3}=-\frac{l}{r^{2}}, \quad \Omega_{v}^{2}=\frac{1}{l} .
$$

Moreover, from the second equation in (1.5), one can find the non-zero components of $h_{\mu}^{a}$ as follows

$$
\begin{aligned}
& h_{r}^{1}=\frac{1-\alpha l^{2} \Lambda_{0}}{2 \mu l r(1+\sigma \alpha)^{2}}, \quad h_{u}^{3}=\frac{1-\alpha l^{2} \Lambda_{0}}{2 \mu r^{2}(1+\sigma \alpha)^{2}}, \quad h_{v}^{2}=\frac{1-\alpha l^{2} \Lambda_{0}}{2 \mu l^{2}(1+\sigma \alpha)^{2}}, \\
& h_{u}^{2}=-\frac{1}{4 \mu l^{2}(1+\sigma \alpha)^{2}}\left(-2 r^{2} \frac{\partial^{2} F}{\partial r^{2}}+2 r \frac{\partial F}{\partial r}+\left(\alpha l^{2} \Lambda_{0}-1\right) F\right),
\end{aligned}
$$


where

$$
\Lambda_{0}=\frac{1}{\alpha l^{2}}+\frac{2 \mu^{2}(1+\sigma \alpha)^{3}}{\alpha^{3}}\left(1 \mp \sqrt{1+\frac{\alpha^{2}}{\mu^{2} l^{2}(1+\sigma \alpha)^{2}}}\right) .
$$

Plugging everything into the equation (1.6), for $1+\sigma \alpha \neq 0$, one arrives at ${ }^{6}$

$$
-r^{2} \frac{\partial^{3} F}{\partial r^{3}}-\frac{\mu l}{\alpha}\left[(1+\sigma \alpha)^{2} \mp \sqrt{(1+\sigma \alpha)^{2}+\frac{\alpha^{2}}{\mu^{2} l^{2}}}\right]\left(r \frac{\partial^{2} F}{\partial r^{2}}-\frac{\partial F}{\partial r}\right)=0,
$$

which is exactly the same as (3.5), taking into account the following identity

$$
\tilde{\mu}=-\frac{\mu}{\alpha}\left[(1+\sigma \alpha)^{2}-\sqrt{(1+\sigma \alpha)^{2}+\frac{\alpha^{2}}{\mu^{2} l^{2}}}\right] .
$$

\section{On new MMG vacuum solutions}

It is known that any Einstein solution is a solution of TMG and since locally MMG has the same structure as that of TMG, it should also be a solution of MMG. However, TMG has more solutions than just the Einstein solutions and therefore it is natural to expect that MMG should also have more solutions than Einstein or even TMG itself.

In particular one can see that for every value of $\mu l \neq 3$ in TMG, there are vacuum solutions with $\mathrm{SL}(2, \mathbb{R}) \times \mathrm{U}(1)$ isometry known as warped AdS geometries [4-7] (see also [17] for NMG). The U(1) factor in the isometry group could be timelike or spacelike. Recently it has been shown [18] that the MMG model also admits warped AdS solutions. More precisely, starting with the following ansatz

$$
d s^{2}=\frac{l^{2}}{\nu^{2}+3}\left[-\cosh ^{2} \rho d \tau^{2}+d \rho^{2}+\frac{4 \nu^{2}}{\nu^{2}+3}(d \xi+\sinh \rho d \tau)^{2}\right]
$$

or

$$
d s^{2}=\frac{l^{2}}{\nu^{2}+3}\left[\cosh ^{2} \rho d \xi^{2}+d \rho^{2}-\frac{4 \nu^{2}}{\nu^{2}+3}(d \tau+\sinh \rho d \xi)^{2}\right]
$$

it can be shown that they satisfy the equations of motion (1.7) if

$$
\begin{aligned}
\bar{\Lambda}_{0} & =-\frac{1}{4 \mu^{2} l^{4}}\left[4 \mu^{2} l^{2} \bar{\sigma} \nu^{2}+12 \mu l\left(\nu^{3}-\nu\right)+\gamma\left(-8 \nu^{4}+18 \nu^{2}-9\right)\right], \\
\tilde{\mu} l+3 \nu & =\left(\nu^{2}-1\right) \frac{\gamma}{\mu l}
\end{aligned}
$$

which can be solved to find $\nu$ and $l$ it terms of the parameters of the model.

For $\nu=1$ where $\tilde{\mu} l=-3$, the equations of motion admit a null warped AdS solution as follows

$$
d s^{2}=l^{2}\left(\frac{d r^{2}}{r^{2}}+\frac{d u d v}{r^{2}}+\frac{d^{2} u}{r^{4}}\right), \quad \text { with } \quad l^{2}=-\frac{1}{2 \bar{\Lambda}_{0}}\left(\bar{\sigma}+\frac{\tilde{\mu}}{\mu}\right)
$$

which is indeed the Schrödinger solution we have found in the previous section with the dynamical scaling $z=2$.

\footnotetext{
${ }^{6}$ Note that in order to get a well defined limit at $\alpha=0$ one needs to choose the upper sign.
} 
Note that in the $\gamma \rightarrow 0$ limit, the above solution reduces to that of TMG and, in particular we get $3 \nu=-\mu l$. One may also consider a limit in which the coefficient of the Cotton tensor vanishes, though the coefficient of the curvature-squared symmetric tensor $J$ survives in this limit. This limit might be taken as $\mu \rightarrow \infty, \gamma \rightarrow \infty$ while keeping $\kappa=\frac{\gamma}{\mu^{2}}$ fixed. In this limit one still gets the warped AdS solution with

$$
\bar{\Lambda}_{0}=\frac{\bar{\sigma}^{2}}{\kappa}, \quad l^{2}=\left(\nu^{2}-\frac{3}{2}\right) \frac{\bar{\sigma}}{\bar{\Lambda}_{0}} .
$$

It is easy to show that the warped AdS geometry with the above parameters solves the following equations of motion

$$
\bar{\sigma} G_{\mu \nu}+\bar{\Lambda}_{0} g_{\mu \nu}+\kappa J_{\mu \nu}=0,
$$

which can be obtained from the equations of motion of the MMG model by setting the coefficient of the Cotton tensor to zero. One could also show that the above equations of motion admit several vacuum solutions including AdS, warped AdS, null warped AdS and AdS-wave solutions. It is then natural to wonder whether this equation could also define a consistent three dimensional gravity. We note, however, that to have a consistent theory the Bianchi identity should also be satisfied. ${ }^{7}$

Actually from the equation (4.6) and taking into account that the Bianchi identity is satisfied for the Einstein tensor one gets $D_{\mu} J^{\mu \nu}=0$. On the other hand from a direct computation one has [1]

$$
\sqrt{-g} D_{\mu} J^{\mu \nu}=\epsilon^{\nu \rho \sigma} S_{\rho}^{\tau} C_{\sigma \tau}, \quad \text { with } \quad S_{\mu \nu}=R_{\mu \nu}-\frac{1}{4} g_{\mu \nu} R .
$$

To make the theory consistent one should set the right hand side of the above equation to zero

$$
\epsilon^{\nu \rho \sigma} S_{\rho}^{\tau} C_{\sigma \tau}=0
$$

for all $\nu$. Therefore the equation (4.6) may define a consistent three dimensional gravity if the above constraint holds. This constraint guarantees that the Bianchi identity is satisfied on shell. It is easy to see that the constraint (4.8) is satisfied by the warped AdS solution (4.5).

To find further consistent solutions of the model defined by the equation (4.6) consider the following ansatz for a black hole solution

$$
d s^{2}=\frac{l^{2}}{r^{2}}\left(-f(r) d t^{2}+\frac{d r^{2}}{f(r)}+d x^{2}\right) .
$$

Plugging this ansatz into the constraint equation (4.8) one finds

$$
f(r)=1+b_{0} r+b_{1} r^{2},
$$

where $b_{0}$ could be zero. Indeed when $b_{0}$ is zero the ansatz is a solution of the equation (4.6) if

$$
\bar{\Lambda}_{0}=-\frac{\kappa+4 l^{2} \bar{\sigma}}{4 l^{4}} .
$$

\footnotetext{
${ }^{7}$ We would like to thank the referee for his/her comment on this point.
} 
On the other hand if one requires $b_{0} \neq 0$, then the above ansatz is a solution at a particular point $^{8}$ in the moduli space of the parameters of the model

$$
\bar{\Lambda}_{0}=-\frac{\bar{\sigma}}{2 l^{2}}, \quad \kappa=-2 l^{2} \bar{\sigma} .
$$

Note that since the constraint (4.8) is independent of the parameters of the model and taking into account that the Cotton tensor for these solutions is identically zero, the above solutions are also solutions of the equations of motion of MMG. It is also straightforward to see that in the merger point the equation (4.6) admits a null solutions, though in this case since the Cotton tensor is non-zero the corresponding null solution cannot be a solution of MMG. Of course as we have seen the MMG model has its own null solution, though it occurs at another point in the moduli space of the parameters.

The model also admits the wave solution

$$
d s^{2}=\frac{l^{2}}{r^{2}}\left(d r^{2}-2 d u d v-F(r) d u^{2}\right),
$$

for which the constraint (4.8) is satisfied identically. From the equation (4.6) one finds $F(r)=1+c r^{2}$ if the parameters of the model satisfy the equation (4.11). It is worth noting that at the merger point (4.12) both the constraint and the equation of motion (4.6) are satisfied identically by the wave metric (4.13) for arbitrary function $F$. In other words the function $F$ cannot be fixed by the equation of motion. We should admit that the significant of this solution is not clear to us. It would be interesting to explore this point better. Note that this is not the case for MMG where at this point the function $F$ is fixed by the corresponding equations of motion to be $F=1+c_{0} r+c_{1} r^{2}$.

To conclude we note that the equation (4.6) together with the constraint (4.8) may define a consistent three dimensional gravity. Note that since the resulting mode has several non-trivial vacuum solutions besides that of an AdS, it should not be equivalent to three dimensional Einstein gravity.

\section{Logarithmic CFT and new anomaly}

In sections two and three we have found that for particular values of the parameters of the MMG model, it can admit logarithmic solutions. In the case of the TMG model it was conjectured [9] that when the logarithmic modes appear, the dual field theory would be a LCFT with one zero central charge [19, 20]. For the NMG model [21] the situation is the same though both the central charges are zero (see for example [22, 23]).

When the central charge is zero, the two point function of the corresponding energy momentum tensor should vanish as well. We note, however, that in a LCFT one has the logarithmic partner of stress-tensor which could have non-zero two point function whose expression is fixed by a new parameter known as "new anomaly". Since the local structure of MMG is the same as TMG, one would expect to see the same behavior for MMG too. Indeed this is the aim of this section to evaluate the new anomaly for MMG model.

\footnotetext{
${ }^{8}$ This point was called merger point in [18].
} 
To proceed let us recall that the asymptotic symmetry algebra of AdS geometry in MMG model consists of two copies of the Virasoro algebra with central charges [1]

$$
c_{ \pm}=\frac{3 l}{2 G}\left(\sigma \pm \frac{1}{\mu l}+\frac{\alpha-\alpha^{2} \Lambda_{0} l^{2}}{2 \mu^{2} l^{2}(1+\sigma \alpha)^{2}}\right) .
$$

Note that in the TMG limit $\alpha \rightarrow 0$ the above central charges reduce to the that of TMG. By making use of the equation (3.13), the expression for central charges may be recast into the following form

$$
c_{ \pm}=\frac{3}{2 \alpha \mu G}\left(\sqrt{\alpha^{2}+\mu^{2} l^{2}(1+\sigma \alpha)^{2}}-\mu l \pm \alpha\right) .
$$

It is then straightforward to evaluate the value of the central charges at the critical point $\tilde{\mu} l=-1$ where the corresponding geometry could provide a holographic description for a LCFT

$$
\tilde{\mu} l=-1:\left[\text { or } \alpha=-2\left(\sigma-\frac{1}{\mu l}\right)\right] \rightarrow c_{+}=\frac{3}{\mu G}, \quad c_{-}=0 .
$$

As we see the central charge of the left moving sector is zero and therefore one should naturally consider a new mode corresponding to the logarithmic partner of the energy momentum tensor whose two point correlation function would be fixed by the new anomaly. Following $[25,26]$ the new anomaly can be evaluated as follows

$$
b_{L} \equiv b_{-}=\lim _{\alpha \rightarrow \alpha_{c}} \frac{c_{-}}{h^{(0)}-h^{(m)}},
$$

where $\alpha_{c}=-2\left(\sigma-\frac{1}{\mu l}\right)$. Note that in this limit both the denominator and the numerator vanish, though their ratio remains finite and nonzero. More precisely, from (2.15) and using the identity (3.15) one gets

$$
h^{(0)}-h^{(m)}=\frac{1}{2 \alpha}\left(\alpha-\mu l(1+\sigma \alpha)^{2}+\sqrt{\alpha^{2}+\mu^{2} l^{2}(1+\sigma \alpha)^{2}}\right) .
$$

Plugging this expression into the equation (5.4) and performing the limit $\alpha \rightarrow \alpha_{c}$ one arrives at

$$
b_{-}=-\frac{3 l}{G} \frac{\mu l}{(\mu l \sigma-2)^{2}} .
$$

Clearly in the TMG limit where $\sigma=1, \mu l=1$ one has

$$
c_{+}=\frac{3 l}{G}, \quad b_{-}=-\frac{3 l}{G}, \quad c_{-}=0,
$$

in agreement with the results of $[19,20]$.

Therefore denoting by $(T(z), \bar{T}(\bar{z}))$ and $(t(z), \bar{t}(\bar{z}))$ the energy momentum tensor and its logarithmic partner, respectively, it is natural to expect that the three dimensional MMG gravity at the critical point would provide a holographic dual for a LCFT such that

$$
\begin{aligned}
\langle T(z) T(0)\rangle & =\frac{c_{-}}{2 z^{4}}=0, \quad\langle\bar{T}(\bar{z}) \bar{T}(0)\rangle=\frac{3 l / G}{2 \bar{z}^{4}}, \quad\langle T(z) t(0)\rangle=\frac{\mu l}{(\mu l \sigma-2)^{2}} \frac{-3 l / G}{2 z^{4}}, \\
\langle t(z) t(0)\rangle & =\frac{3 l}{G} \frac{\mu l}{(\mu l \sigma-2)^{2}} \frac{\ln \left(m^{2} z^{2}\right)}{z^{4}},
\end{aligned}
$$

where $m$ is a scale. It would be interesting to derive the above equation using the holographic renormalization procedure (see for example [19] for TMG and [23] for NMG). 


\section{Conclusions}

In this paper using the metric formulation of the MMG model we have studied linearized equations of motion around an AdS vacuum solution. We have seen that the model has locally the same structure as that of the TMG model. More precisely, in the linearized level, upon a redefinition of the parameters, the corresponding equations of motion exactly reduce to that of TMG and therefore both models have the same spectrum. In particular there are critical points at which the massive mode degenerates with the massless graviton. Of course there is big difference between MMG and TMG in the sense that although in TMG one is forced to be at the critical points, a priori, there is no such requirement in MMG. In fact as it was shown in [1] there is a wide range of the parameters where the model would be well defined.

Indeed, although the resulting equations for ther perturbations around an AdS vacuum are the same for both TMG and MMG models, the two models have different conserved charges. This is indeed the reason why one could get a non-trivial well defined 3D gravity from MMG model. Actually, since in the metric formulation of MMG one has no action, it is tricky to find the conserved charges of the model.

We note, however, that even though one cannot write an action for MMG in the metric formulation, since in the first order of perturbation, the resulting equations are exactly the same as that in TMG, one would expect that, at least, at second order in perturbation it is possible to write an action whose equations of motion are the corresponding linearized equations. Therefore it should be possible to read off the energy of different modes using the results of TMG. In particular from equations (70)-(72) of [8], up to numerical factors, one gets

$$
\begin{aligned}
E_{M} & \sim \frac{M^{2}}{\mu G} \int d^{3} x \sqrt{-\bar{g}} \epsilon_{\beta}^{0 \mu} h_{M}^{\beta \nu} \dot{h}_{M \mu \nu}, \\
E_{L} & \sim-\frac{c_{-}}{l G} \int d^{3} x \sqrt{-\bar{g}} \bar{\nabla}^{0} h_{L}^{\mu \nu} \dot{h}_{L \mu \nu}, \\
E_{R} & \sim-\frac{c_{+}}{l G} \int d^{3} x \sqrt{-\bar{g}} \bar{\nabla}^{0} h_{R}^{\mu \nu} \dot{h}_{R \mu \nu} .
\end{aligned}
$$

Here we have used a decomposition by which $h_{\mu \nu}=h_{M \mu \nu}+h_{L \mu \nu}+h_{R \mu \nu}$ and also $M^{2}$ and $c_{ \pm}$are given by (2.10) and (5.1), respectively.

We have also found AdS wave solutions of the model and shown that at the critical points the model exhibit logarithmic solutions. Therefore at the critical point the model could provide a holographic dual for a LCFT. We have also calculated the new anomaly of the theory.

We have observed that in the metric formulation of MMG if one sets the coefficient of the Cotton tensor to zero, it might still define a new consistent three dimensional gravity. Indeed a consistency condition requires to have the constraint $\epsilon^{\nu \rho \sigma} S_{\rho}^{\tau} C_{\sigma \tau}=0$ as well.

It is important to mention that since the resultant model has several vacuum solutions besides the AdS one, it should not be equivalent to three dimensional Einstein gravity. Even though it has a common feature with 3D conformal gravity, since the model has a null solution it cannot be equivalent to three dimensional conformal gravity either. We note also that since the constraint (4.8) is independent of the parameters appearing in 
the equations of motion, any solution of the MMG model is a solution of the new model defined by the equation (4.6). Though the other way around is not correct. In particular at the merger point the new model has the wave solution (4.13) with arbitrary $F$, though in MMG the function $F$ is fixed.

In the Chern-Simons like formalism of the MMG model setting the coefficient of the Chern-Simons term to zero one arrives at ${ }^{9}$

$$
L=-\sigma e \cdot R+\frac{\Lambda_{0}}{6} e \cdot e \times e+h \cdot T(\omega)+\frac{\alpha}{2} e \cdot h \times h,
$$

which as pointed out in [1] should be equivalent to the Einstein gravity. It is, however, important to note that in general the equations of motion obtained from this action cannot be mapped to that defined by the equation (4.6). Therefore there is no conflict between the results of our paper with the fact that the model (6.2) could be equivalent to the Einstein gravity.

To further explore the properties of MMG it would be interesting to study holographic renormalization of the model. We note, however, that since in this context the action plays an essential role one will have to work with the Chern-Simons like formulation of the model.

Note added. When we were in the final stage of submitting our paper, the paper [27] appeared in the arXiv which has some overlap with ours.

\section{Acknowledgments}

We would like to thank Hamid Afshar and Mahmoud safari for useful discussions. M. M. Q. also thanks Farhad Ardalan, Hessamaddin Arfaei and Shahin Rouhani for encouragement and support.

Open Access. This article is distributed under the terms of the Creative Commons Attribution License (CC-BY 4.0), which permits any use, distribution and reproduction in any medium, provided the original author(s) and source are credited.

\section{References}

[1] E. Bergshoeff, O. Hohm, W. Merbis, A.J. Routh and P.K. Townsend, Minimal Massive 3D Gravity, Class. Quant. Grav. 31 (2014) 145008 [arXiv:1404.2867] [INSPIRE].

[2] S. Deser, R. Jackiw and S. Templeton, Topologically Massive Gauge Theories, Annals Phys. 140 (1982) 372 [Erratum ibid. 185 (1988) 406] [INSPIRE].

[3] O. Hohm, A. Routh, P.K. Townsend and B. Zhang, On the Hamiltonian form of 3D massive gravity, Phys. Rev. D 86 (2012) 084035 [arXiv:1208.0038] [INSPIRE].

[4] K.A. Moussa, G. Clement and C. Leygnac, The black holes of topologically massive gravity, Class. Quant. Grav. 20 (2003) L277 [gr-qc/0303042] [INSPIRE].

[5] A. Bouchareb and G. Clement, Black hole mass and angular momentum in topologically massive gravity, Class. Quant. Grav. 24 (2007) 5581 [arXiv:0706.0263] [INSPIRE].

[6] D. Anninos, W. Li, M. Padi, W. Song and A. Strominger, Warped AdS $S_{3}$ Black Holes, JHEP 03 (2009) 130 [arXiv:0807.3040] [INSPIRE].

\footnotetext{
${ }^{9}$ Note that to maintain $\gamma \rightarrow \infty$ one has to set $\sigma \alpha \rightarrow-1$.
} 
[7] K.A. Moussa, G. Clement, H. Guennoune and C. Leygnac, Three-dimensional Chern-Simons black holes, Phys. Rev. D 78 (2008) 064065 [arXiv:0807.4241] [INSPIRE].

[8] W. Li, W. Song and A. Strominger, Chiral Gravity in Three Dimensions, JHEP 04 (2008) 082 [arXiv:0801.4566] [INSPIRE].

[9] D. Grumiller and N. Johansson, Instability in cosmological topologically massive gravity at the chiral point, JHEP 07 (2008) 134 [arXiv:0805.2610] [INSPIRE].

[10] M. Alishahiha and R. Fareghbal, D-Dimensional Log Gravity, Phys. Rev. D 83 (2011) 084052 [arXiv:1101.5891] [InSPIRE].

[11] I. Gullu, M. Gurses, T.C. Sisman and B. Tekin, AdS Waves as Exact Solutions to Quadratic Gravity, Phys. Rev. D 83 (2011) 084015 [arXiv:1102.1921] [InSPIRE].

[12] E.A. Bergshoeff, O. Hohm, J. Rosseel and P.K. Townsend, Modes of Log Gravity, Phys. Rev. D 83 (2011) 104038 [arXiv:1102.4091] [INSPIRE].

[13] N. Johansson, A. Naseh and T. Zojer, Holographic two-point functions for 4d log-gravity, JHEP 09 (2012) 114 [arXiv:1205.5804] [INSPIRE].

[14] E. Ayon-Beato and M. Hassaine, pp waves of conformal gravity with self-interacting source, Annals Phys. 317 (2005) 175 [hep-th/0409150] [INSPIRE].

[15] E. Ayon-Beato and M. Hassaine, Exploring AdS waves via nonminimal coupling, Phys. Rev. D 73 (2006) 104001 [hep-th/0512074] [INSPIRE].

[16] G. Clement, Black holes with a null Killing vector in new massive gravity in three dimensions, Class. Quant. Grav. 26 (2009) 165002 [arXiv:0905.0553] [INSPIRE].

[17] G. Clement, Warped $A d S_{3}$ black holes in new massive gravity, Class. Quant. Grav. 26 (2009) 105015 [arXiv:0902 .4634] [INSPIRE].

[18] A.S. Arvanitakis, A.J. Routh and P.K. Townsend, Matter coupling in 3D "Minimal Massive Gravity", arXiv:1407.1264 [INSPIRE].

[19] K. Skenderis, M. Taylor and B.C. van Rees, Topologically Massive Gravity and the AdS/CFT Correspondence, JHEP 09 (2009) 045 [arXiv: 0906.4926] [INSPIRE].

[20] D. Grumiller and I. Sachs, $A d S_{3} / L C F T_{2}$ - Correlators in Cosmological Topologically Massive Gravity, JHEP 03 (2010) 012 [arXiv:0910.5241] [INSPIRE].

[21] E.A. Bergshoeff, O. Hohm and P.K. Townsend, Massive Gravity in Three Dimensions, Phys. Rev. Lett. 102 (2009) 201301 [arXiv:0901.1766] [INSPIRE].

[22] D. Grumiller and O. Hohm, AdS $S_{3} / L C F T_{2}$ : Correlators in New Massive Gravity, Phys. Lett. B 686 (2010) 264 [arXiv:0911.4274] [InSPIRE].

[23] M. Alishahiha and A. Naseh, Holographic renormalization of new massive gravity, Phys. Rev. D 82 (2010) 104043 [arXiv: 1005.1544] [INSPIRE].

[24] Y. Kwon, S. Nam, J.-D. Park and S.-H. Yi, Quasi Normal Modes for New Type Black Holes in New Massive Gravity, Class. Quant. Grav. 28 (2011) 145006 [arXiv:1102.0138] [INSPIRE].

[25] D. Grumiller, N. Johansson and T. Zojer, Short-cut to new anomalies in gravity duals to logarithmic conformal field theories, JHEP 01 (2011) 090 [arXiv: 1010.4449] [INSPIRE].

[26] D. Grumiller, W. Riedler, J. Rosseel and T. Zojer, Holographic applications of logarithmic conformal field theories, J. Phys. A 46 (2013) 494002 [arXiv:1302.0280] [INSPIRE].

[27] B. Tekin, Minimal Massive Gravity: Conserved Charges, Excitations and the Chiral Gravity Limit, Phys. Rev. D 90 (2014) 081701 [arXiv:1409.5358] [INSPIRE]. 